\title{
18. ACOUSTIC PROPERTIES OF ALTERED PERIDOTITE AT SITE 637 FROM LABORATORY AND SONIC WAVEFORM DATA ${ }^{1}$
}

\author{
D. Goldberg, Lamont-Doherty Geological Observatory, Palisades, New York \\ and \\ B. Zinszner, Institut Français du Pétrole, 92506 Rueil-Malmaison, France
}

\begin{abstract}
Extensional and shear-wave velocities and attenuations $\left(\mathrm{V}_{e}, \mathrm{~V}_{s}, \mathrm{Q}_{e}\right.$, and $\left.\mathrm{Q}_{s}\right)$ were measured using the resonant bar technique in the same frequency range as used in sonic logging $(10-20 \mathrm{kHz})$. Laboratory measurements at a simulated in-situ effective stress of about $6.3 \mathrm{MPa}$ were made on seven core samples from an interval 230 to $252 \mathrm{~m}$ below seafloor at Site 637. High-frequency $(500-\mathrm{kHz})$ transmission experiments were also conducted on one sample to measure $\mathrm{V}_{p}$ and $\mathrm{Q}_{p}$ under uniaxial compressive stresses up to $16.0 \mathrm{MPa}$. Ultrasonic velocity was $10.3 \%$ greater than resonant bar results, whereas predicted dispersion for a constant-Q model is about $6.6 \%$ over this frequency band. Errors were generally less than $10 \%$ in both experiments, and the large apparent dispersion was probably due to interfering geometric effects in small samples.

Compressional-wave velocity and attenuation were also computed from sonic log $(10-20-\mathrm{kHz})$ waveforms recorded over the same interval in this well. $\mathrm{V}_{p}$ and $\mathrm{Q}_{p}$ were calculated by the arrival-time delay and the spectral ratio, respectively, of compressional waveforms at 2.44- and 3.05-m receiver spacings from the source. The pressure-compensated resonant bar measurements of $V_{p}$ are in good agreement with the sonic log. However, resonant bar measurements of $Q_{p}$ are generally greater than the log-derived values, primarily because extrinsic effects such as interferences and scattering from boundaries and radiation of shear energy away from the borehole cannot be separated from the apparent in-situ attenuation. Extrinsic losses and intrinsic attenuation in this strongly foliated serpentinite are both important, yielding large apparent attenuation of compressional energy in highly altered zones and breccias.
\end{abstract}

\section{INTRODUCTION}

The elastic properties of $i n$-situ materials are routinely determined by industry-standard sonic logging techniques, which provide a measure of the compressional velocity in the 10- to 20$\mathrm{kHz}$ (sonic) frequency band (Pickett, 1963; Arons et al., 1978). The measurement of attenuation from sonic waveforms, however, poses both theoretical and experimental problems, one of the most difficult being the separation of intrinsic (frictional absorption) and extrinsic (scattering) losses affecting the compressional head wave, which are dominated by different active mechanisms, depending on the frequency (Biot, 1956, 1962; Winkler and Nur, 1979; Spencer, 1981; Murphy, 1982; Goldberg et al., 1984b). Laboratory elastic properties are typically measured using pulse transmission techniques at ultrasonic frequencies from 100 to $1000 \mathrm{kHz}$ (Peselnick and Zietz, 1959; Winkler and Plona, 1982) generating wavelengths on the order of centimeters, in contrast to several tens of centimeters at sonic frequencies. This scale difference further complicates the comparison of laboratory and in-situ measurements. A primary goal of this study was to measure elastic properties in the laboratory at the same frequencies as used in logging and to compare measurements to log values.

To achieve this goal, a laboratory resonance method using a 15- to 20-cm-long bar may be used to yield the elastic parameters at sonic frequencies (Wyllie et al., 1962). The strong dependence of the elastic resonance of a bar on the porosity and bulk moduli requires that measurements be made under confining pressure in the laboratory to simulate the overburden stress of the rock in situ (Birch and Bancroft, 1938; Born, 1941). This paper compares the elastic properties measured from laboratory

${ }^{1}$ Boillot, G., Winterer, E. L., et al., 1988. Proc. ODP, Sci. Results, 103: College Station, TX (Ocean Drilling Program). samples and logging data in altered peridotite drilled at Hole 637A.

At Site $637,74 \mathrm{~m}$ of foliated peridotite was penetrated from 212 to $285.6 \mathrm{~m}$ below seafloor (mbsf). The peridotite is clinopyroxene-bearing spinel harzburgite, which is more than $90 \%$ serpentinized and is pervasively cut by veins of calcite and serpentine ("Site 637" chapter; Shipboard Scientific Party, 1987). In Figure 1, a photomicrograph of a sample from $230.4 \mathrm{mbsf}$ depicts a calcite vein (top) cutting matrix serpentinite. The interparticular spaces were filled by epoxy film polymerized in the pore after vacuum injection as a liquid. Microfractures were filled and are visible as dark lines crosscutting the matrix. The porosity of this sample is $18 \%$.

The interval that was both logged and cored is only $22 \mathrm{~m}$ thick (230-252 mbsf). The most homogeneous rock in this interval was identified and sampled at seven unequally spaced depths, based on the results of core inspection. Homogeneity at the scale of the sample length $(15-20 \mathrm{~cm})$ was impossible, because of the intense alteration and foliation that varied throughout the sample at a scale of 0.1 to $0.5 \mathrm{~cm}$. However, bulk porosities varied only slightly, between $18 \%$ and $19 \%$, for all seven samples. The fragility of these foliated rocks resulted in a small number of prepared samples which had similar characteristics. Unfortunately, this limited the correlation of acoustic properties to changes in porosity and depth.

\section{RESONANT BAR MEASUREMENTS}

The resonant bar technique permits the measurement of elastic velocities and attenuations in the frequency band from 0.5 to $25 \mathrm{kHz}$, subject to the practical limitations of excitation for large samples and geometric errors in small bars. The technique consists of submitting a rock bar, held at its center, to a sinusoidal force at one end and measuring the vibrations at the other. The resonant frequencies of the bar are a function of the velocity of the material as well as the length of the bar; damping of 


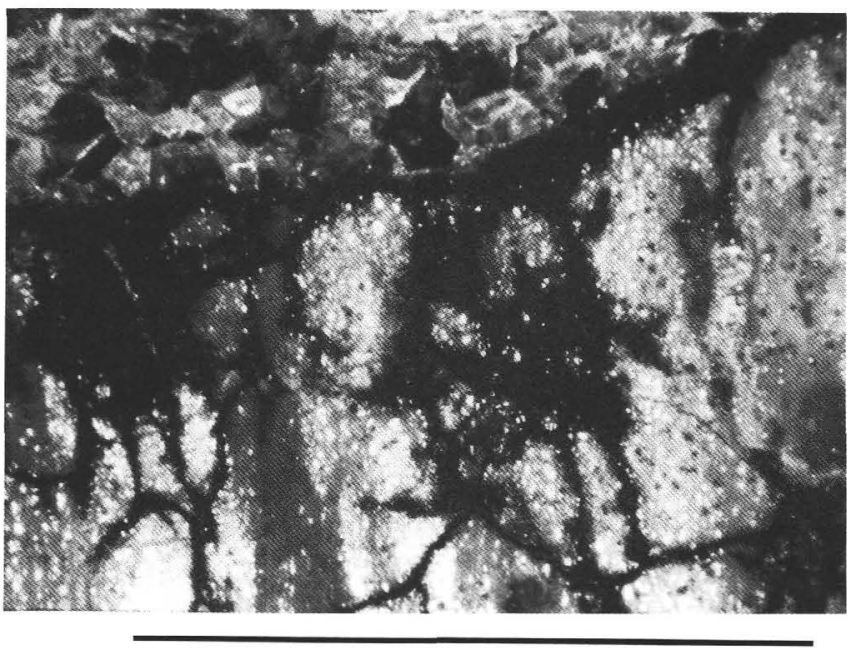

$1 \mathrm{~cm}$

Figure 1. Photomicrograph of altered peridotite from $230.4 \mathrm{mbsf}$, Hole $637 \mathrm{~A}$. Interparticular pore spaces were filled with epoxy after vacuum injection as a liquid. Calcite veins and strong foliation are visible in this sample. The bulk porosity was measured at $18 \%$.

the vibrations near the resonant peak characterizes the attenuation.

The theory and experimental design of the resonant bar technique have been previously discussed in detail by numerous authors (Born, 1941; Wyllie et al., 1962; Winkler, 1979; Murphy, 1982). Three types of modes can be excited: the longitudinal mode, where the bar endures a force of extension and contraction; the torsional mode, where a shear force is applied to the bar; and the bending mode. The extensional and shear modes were independently recorded; the bending mode was not studied because it travels at the same velocity as the extensional mode. Forced and free oscillations of the bar were measured using a light $(0.7-\mathrm{g})$ accelerometer attached with wax, rather than a displacement sensor. The measurement of acceleration introduces a factor of $\omega^{2}$ in the wave equation solution, which strongly weighted higher order resonant peaks having low displacement magnitude. This enabled the visualization of several harmonics, permitting observation of velocity dispersion with increasing frequency; however, higher order modes are more sensitive to anisotropy in the sample and asymmetry in the resonant peaks, which distort or obscure the measurement. Therefore, only the fundamental extensional and shear resonances of the system were studied: $\mathrm{V}_{e}$ and $\mathrm{V}_{s}$ were calculated, and $\mathrm{Q}_{e}$ and $\mathrm{Q}_{s}$ were measured by using both the resonant peak width of forced oscillations and the decay of free vibrations. The two methods usually agreed within $2 \%$.

To approximate the acoustic behavior of rock samples under in-situ conditions, the experiment was conducted at room temperature in a high-pressure vessel, and the samples were fully saturated with distilled water. Slim bars (cross-sectional area of about $2.3 \mathrm{~cm}^{2}$ ) were cut from the cores and jacketed using heatshrink tubing to allow for pore pressures to be introduced and maintained isolated from the confining pressure. An aluminum pipette (about $2 \mathrm{~mm}$ in diameter) was inserted about $5 \mathrm{~mm}$ into the bar at its center of mass, and the samples were placed under vacuum and saturated by pumping distilled water through the pipette. For these low-permeability samples, the pipette method was often ineffective, and sections of the jacket had to be cut out to allow for easier flow through an enlarged surface opening and resealed with rubber tape and silicon gel.

Small bars having little mass and moment of inertia require the greatest proportional corrections for the total mass added to the system; the corrections were as large as $8 \%$ of the measured frequency for these bars and always reduced the apparent velocities and attenuations. An additional correction for lateral extension of the bar was assumed negligible for resonances of low order because a one-dimensional approximation is sufficient for slenderness ratios (length/diameter) of 10 or greater (Schreiber et al., 1973; Bourbié et al., 1987). Measurement effects resulting from induced inertial motions between the pore fluid and the rock in low-permeability systems can also be neglected (Biot, 1956; White, 1986). Our control tests on aluminum and plexiglass bars indicated that measurements were reproducible to within $10 \%$.

For each sample, the recording of the fundamental resonant frequency, $\omega_{0}$, enabled the calculation of $\mathrm{V}_{e}$ and $\mathrm{V}_{s}$ using the expression $\mathrm{V}=\omega_{0} \mathrm{~d} / \pi$, where $\mathrm{d}$ is length of the bar; $\mathrm{Q}_{e}$ and $\mathrm{Q}_{s}$ were calculated using $\Delta \omega / \omega_{0}$, where $\Delta \omega$ is the resonant peak width. Sample lengths of about $15 \mathrm{~cm}$ yielded resonant frequencies in the 5- to $20-\mathrm{kHz}$ frequency band, near to that used in sonic logging. $\mathrm{V}_{e}, \mathrm{~V}_{s}, \mathrm{Q}_{e}, \mathrm{Q}_{s}$, and Poisson's ratio, $\nu$, are presented in Table 1 for seven samples of Site 637 altered peridotite at $0-\mathrm{MPa}$ effective pressure. Mean $\mathrm{V}_{e}$ and $\mathrm{V}_{s}$ are 2327 and 1370 $\mathrm{m} / \mathrm{s}$, respectively, which yields $v=0.44$, although four of the seven individual values (in parentheses in Table 1) were unreliable. Mean $\mathrm{Q}_{e}$ and $\mathrm{Q}_{s}$ are both 12.9 and have large variances. These poor results are caused by reopened microfissures and pore spaces at low confining pressure, which exaggerate the attenuation and measurement errors. Measurements above $0-\mathrm{MPa}$ effective pressure are more reliable.

Figure 2 shows a typical recording obtained for a $100 \%$ water-saturated bar over a range of effective pressures from 0 to 7.0 $\mathrm{MPa}$. Resonant peaks are observed at higher frequencies for progressively greater effective pressures. Shear and extensional resonant frequencies for this sample increased from 6814 to $7619 \mathrm{~Hz}$ and from 9955 to $11,714 \mathrm{~Hz}$, respectively. The corresponding changes in extensional velocity, $\mathrm{V}_{e}$, vs. effective pressure are calculated for two samples from the same depth and plotted in Figure 3. Curves were drawn to illustrate the similar pressure dependence, but there is nearly $10 \%$ difference in the magnitude of $\mathrm{V}_{e}$ in adjoining samples. Because measurement errors in $\mathrm{V}_{s}$ for these small, inhomogeneous samples were large, $\nu$ and the attendant $\mathrm{V}_{p}$ computed by $\mathrm{V}_{p}{ }^{2}=\mathrm{V}_{e}{ }^{2}(1-\nu) /[(1-$ $2 \nu)(1+\nu)]$ were often large. The most reliable measurements of $\mathrm{V}_{e}$ and $\mathrm{V}_{s}$ from this sampling depth were averaged to give a representative $\nu=0.345$ over the entire interval. The resulting $\mathrm{V}_{p}$ calculations and measured $\mathrm{Q}_{e}$ values at 0,5 , and $7.5 \mathrm{MPa}$ are presented for six of the seven samples in Table 2. In general, $\mathbf{Q}_{e}$ is greater than $\mathrm{Q}_{s}$, and $\mathrm{Q}_{e}$ approaches $\mathrm{Q}_{s}$ as pressure increases. $\mathrm{Q}_{p}$ can be assumed to be equal to or slightly greater than $\mathrm{Q}_{e}$ at elevated pressures by the theoretical inequality relationship $\mathrm{Q}_{p}$ $\geq \mathrm{Q}_{e} \geq \mathrm{Q}_{s}$ (Winkler and Nur, 1979). At 6.3-MPa effective pressure, the approximate overburden pressure equivalent at 240 mbsf, mean values for six measurements of $\mathrm{V}_{p}$ and $\mathrm{Q}_{e}$ are $3467 \mathrm{~m} / \mathrm{s}$ and $23(\sigma=278 \mathrm{~m} / \mathrm{s}$ and 1.5$)$, respectively.

\section{ULTRASONIC MEASUREMENTS}

To study the pressure sensitivity of compressional velocity and attenuation, an additional study was conducted under uniaxial compression by ultrasonic transmission at frequencies near 500 $\mathrm{kHz}$. These measurements were made on cylindrical plugs about $4 \mathrm{~cm}$ long and $2.2 \mathrm{~cm}$ in diameter, fully saturated with distilled water. Wave transmission using piezoelectric transducers, one as emitter and one as receiver on each face of the sample, generated acoustic wavelengths of $1 \mathrm{~cm}$ or less. The sample was placed in a press with a dynamic pressure gauge, and the entire assemblage was submerged in water. When the transmitter/sample coupling was sufficient for a signal to be received and recorded on a digitizing oscilloscope, the compressional-wave ve- 
Table 1. Resonant bar measurements of extensional and shear velocities $\left(\mathbf{V}_{e}, \mathbf{V}_{s}\right)$ and quality factors $\left(\mathbf{Q}_{e}, \mathbf{Q}_{s}\right)$ and the calculated Poisson's ratio $(\nu)$ at 0 -MPa effective pressure for seven samples of altered peridotite at ODP Site 637. The mean and standard deviation $(\sigma)$ are calculated for all measurements.

\begin{tabular}{|c|c|c|c|c|c|}
\hline $\begin{array}{l}\text { Depth } \\
\text { (mbsf) }\end{array}$ & $\begin{array}{c}\mathrm{V}_{e} \\
(\mathrm{~m} / \mathrm{s})\end{array}$ & $\begin{array}{c}\mathrm{V}_{s} \\
(\mathrm{~m} / \mathrm{s})\end{array}$ & $\mathrm{Q}_{e}$ & $\mathrm{Q}_{S}$ & $\nu$ \\
\hline 230.4 & 2332 & 1409 & - & - & 0.37 \\
\hline 230.4 & 2554 & 1571 & 15.2 & 13.6 & 0.32 \\
\hline 232.7 & 2493 & 1392 & 21.5 & 18.5 & $(0.60)$ \\
\hline 234.3 & 2316 & 1363 & 14.0 & 11.2 & 0.44 \\
\hline 238.9 & 1756 & 884 & 9.5 & 11.2 & $(0.97)$ \\
\hline 249.0 & 2047 & 1384 & 10.7 & 9.8 & $(0.09)$ \\
\hline 252.1 & 2788 & 1586 & 6.4 & - & $(0.55)$ \\
\hline mean & 2327 & 1370 & 12.9 & 12.9 & 0.44 \\
\hline$\sigma$ & 341 & 233 & 5.2 & 3.4 & 0.26 \\
\hline
\end{tabular}

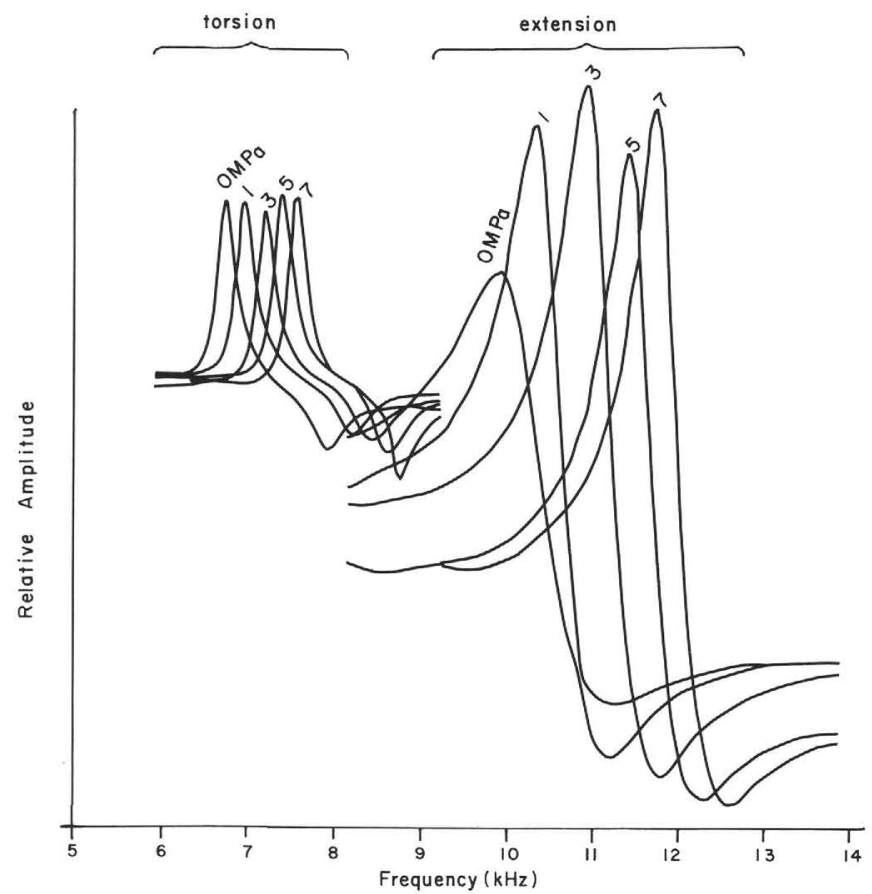

Figure 2. Spectral plot of the first harmonic of torsional and extensional modes at effective pressures of $0,1,3,5$, and $7 \mathrm{MPa}$. Torsional (shear) and extensional wave modes were recorded independently. $\mathrm{V}_{e}, \mathrm{~V}_{s}, \mathrm{Q}_{e}$, and $\mathrm{Q}_{S}$ were measured from these resonance peaks and from the decay envelope of free oscillations. The two methods usually agreed within about $2 \%$.

locity was calculated simply by dividing the height of the sample by the "time-of-flight" between first arrivals of the emitted and received signals. The attenuation was calculated by comparing the frequency spectra of the received signal and a reference signal in a nonattenuating material such as aluminum. The spectral ratio technique has the advantage that the source function and geometrical effects cancel out. $\mathrm{Q}_{p}$ can be assumed to be independent of frequency, such that $\mathrm{Q}_{p}=\omega /(2 \alpha \nu[\omega])$, and $\alpha(\omega)$ can be calculated directly from the spectral ratio (Kjartansson, 1979).

$\mathrm{V}_{p}$ and $\mathrm{Q}_{p}$ were calculated for uniaxial pressures from 0 to 16 $\mathrm{MPa}$. The effect of uniaxial pressure on ultrasonic signals is illustrated in Figure 4. As shown in this example, time-of-flight

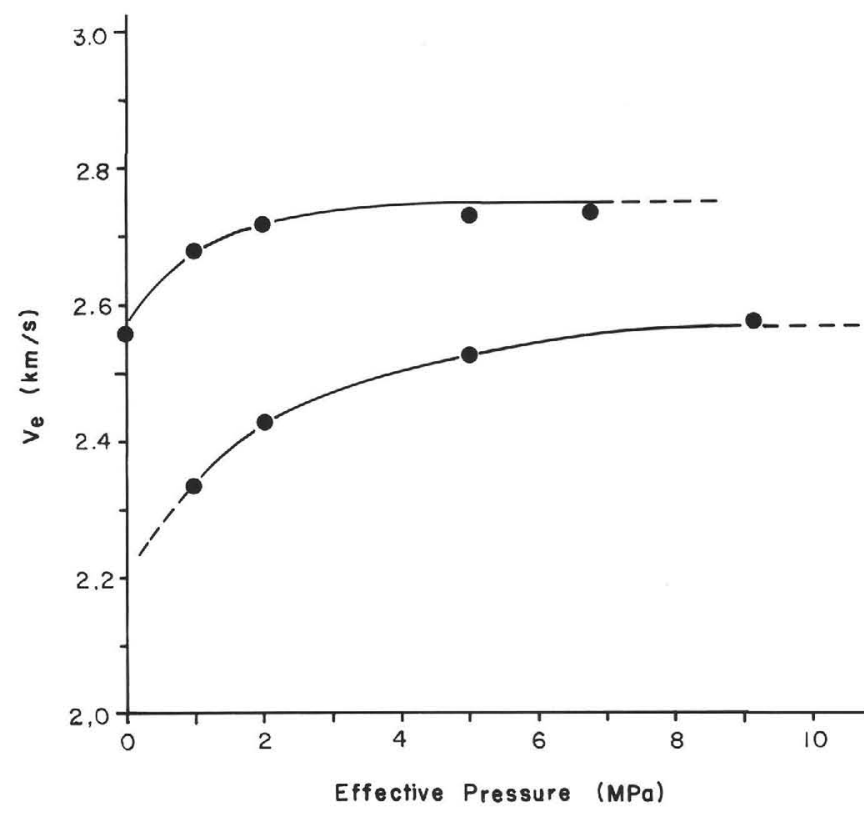

Figure 3. Plot of extensional velocity as a function of effective pressure for two fully saturated samples from $230.4 \mathrm{mbsf}$, Hole 637A. As porosity decreases and elastic moduli increase with increasing effective pressure, $\mathrm{V}_{e}$ also increases. The behavior of the two samples is similar, although the $10 \%$ difference in velocity is indicative of the heterogeneity of altered peridotite at this site.

Table 2. Resonant bar measurements of compressional velocity $\left(\mathrm{V}_{p}\right)$ and quality factor $\left(\mathrm{Q}_{e}\right)$ at $0-, 5-$, and $7.5-$ $\mathrm{MPa}$ effective pressure $\left(\mathbf{P}_{e}\right)$ for seven samples of altered peridotite from ODP Site 637 . The mean and standard deviation $(\sigma)$ are calculated for all measurements.

\begin{tabular}{|c|c|c|c|c|c|c|c|}
\hline \multirow[b]{3}{*}{$\begin{array}{l}\text { Depth } \\
\text { (mbsf) }\end{array}$} & \multirow{2}{*}{$\begin{array}{c}\mathrm{P}_{e} \\
(\mathrm{MPa}) \\
\end{array}$} & \multicolumn{3}{|c|}{$\mathrm{Q}_{e}$} & \multicolumn{3}{|c|}{$\begin{array}{c}\mathrm{V}_{p} \\
(\mathrm{~m} / \mathrm{s})\end{array}$} \\
\hline & & 0 & 5 & 7.5 & 0 & 5 & 7.5 \\
\hline & & & & & & & \\
\hline 230.4 & & - & 23.6 & 26.1 & - & 3165 & 3224 \\
\hline 230.4 & & 15.2 & 21.6 & 24.0 & 3201 & 3415 & 3423 \\
\hline 232.7 & & 21.5 & 24.0 & 25.5 & 3125 & 3195 & 3210 \\
\hline 234.3 & & 14.0 & 22.0 & 25.0 & 2903 & 3260 & 3316 \\
\hline 238.9 & & 9.5 & - & - & 2170 & - & - \\
\hline 249.0 & & 10.7 & 19.0 & 22.7 & 2566 & 3952 & 3972 \\
\hline 252.1 & & 6.4 & 21.0 & 22.7 & 3494 & 3785 & 3815 \\
\hline mean & & $\overline{12.9}$ & 21.9 & $\overline{24.3}$ & $\overline{2893}$ & 3444 & 3475 \\
\hline$\sigma$ & & 4.8 & 1.7 & 1.3 & 405 & 283 & 277 \\
\hline
\end{tabular}

and attenuation increase at progressively lower uniaxial pressures as the elastic moduli decrease. In Figure 5, ultrasonic $\mathrm{V}_{p}$ and $\mathrm{Q}_{p}$ data are shown as a function of uniaxial pressure for a sample from $230.4 \mathrm{mbsf}$; resonant bar results are also shown in Figure 5. For samples from the same depth and at 6.3-MPa effective pressure, measurements of $\mathrm{V}_{p}$ are $10.3 \%$ higher and $\mathrm{Q}_{p}$ about $24 \%$ lower in ultrasonic than in resonant bar (sonic) experiments. The proportional change in velocity predicted by a constant-Q model for dispersion over a limited frequency band is given by $\Delta v=(1 / \pi Q) \times \ln \left(\omega / \omega_{0}\right)$, where $\omega_{0}$ is the reference frequency (Kolsky, 1956; Futterman, 1962). The velocity dispersion over 6 octaves in frequency for $\mathrm{Q}=20$ is $+6.6 \%$. The observed dispersion is probably greater due to interfering amplitude and phase effects from reflections off the sides of the small 


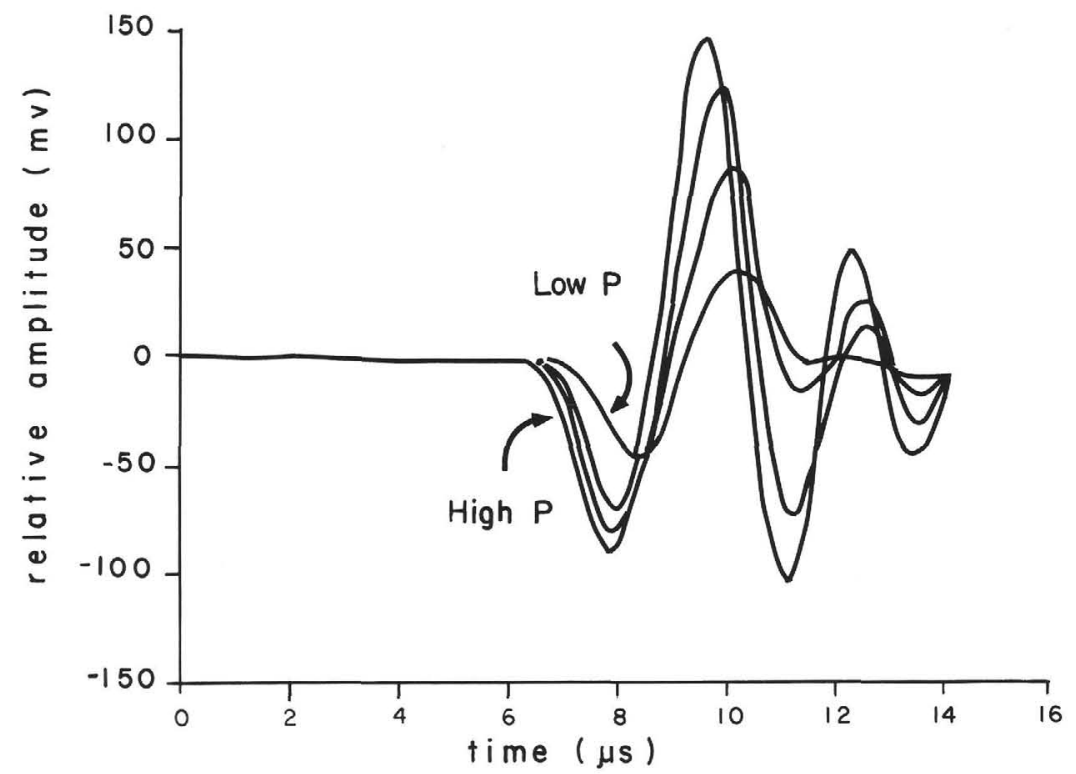

Figure 4. Traces of ultrasonic signals at four arbitrary steps in compression. The relative decreases in signal amplitude and arrival time with decreasing pressure correspond directly with a decrease in velocity and an increase in attenuation as the elastic moduli decrease.

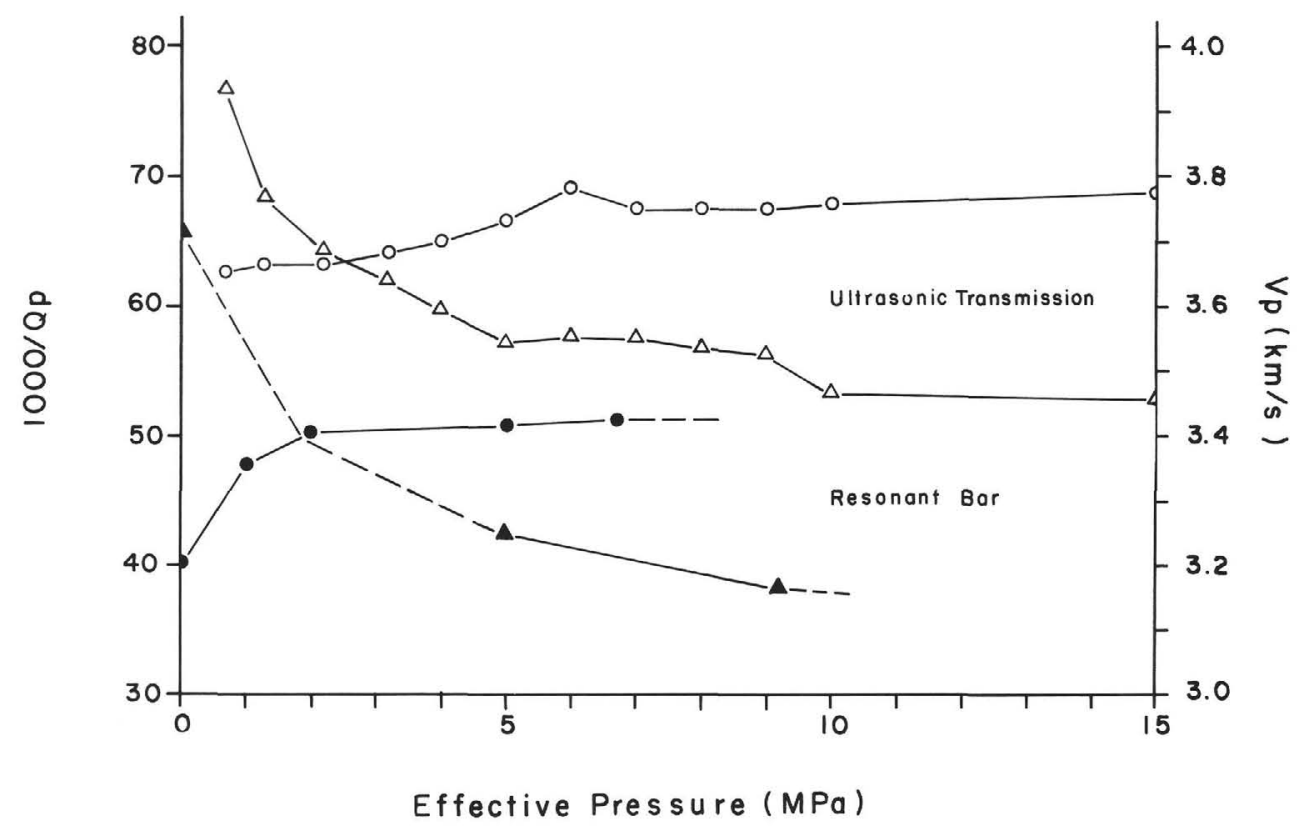

Figure 5. Velocity and attenuation as a function of pressure for ultrasonic and resonant bar measurements on a sample of altered peridotite from $230.4 \mathrm{mbsf}$, Hole 637A. Ultrasonic $\mathrm{V}_{p}$ and $\mathrm{Q}_{p}$ at 6.3-MPa pressure are $3775 \mathrm{~m} / \mathrm{s}$ and 17.5 and resonant bar results are $3421 \mathrm{~m} / \mathrm{s}$ and 23 , respectively, indicating approximately $10.3 \%$ velocity dispersion (see text). The increase in velocity and decrease in attenuation with pressure are similar in both experiments.

sample and by geometric diffraction from the finite length transducer, which influence the absolute values of $\mathrm{V}$ and $\mathrm{Q}(\mathrm{Pa}$ padakis, 1975; Tarif, 1986). However, over a limited increase in pressure, these effects are nearly constant, and the relative changes in $\mathrm{V}$ and $\mathrm{Q}$ are significant. For both resonant bar and ultrasonic transmission tests, $\mathrm{V}_{p}$ increases by $3.2 \%$ and $\mathrm{Q}_{p}$ by about $33 \%$ from 0.7 - to 6.5 -MPa effective pressure. The similar pressure sensitivity for sonic and ultrasonic tests suggests that similar physical mechanisms affect acoustic-wave propagation in both frequency bands.

\section{LOGGING MEASUREMENTS}

The sonic waveform log at Site 637 offers a continuous record of the in-situ physical properties of altered peridotite in this $22-\mathrm{m}$ depth interval, $25 \%$ of which was missed during coring. The Schlumberger long-spaced sonic sonde used to acquire the sonic waveform data operates typically with peak energy in the $10-25-\mathrm{kHz}$ frequency band. A pressure pulse in the borehole fluid generates critically refracted head waves propagating in the formation as well as a series of borehole modes. Paillet and 
White (1982) fully discussed the propagation of acoustic waves in boreholes. Formation velocity is typically calculated from the delay time between refracted head waves back in the borehole fluid at receivers separated by a known distance. Wave amplitudes depend primarily on the coupling of energy at the borehole wall and the distance traveled between source and receiver, but they also depend in part on the dissipation of energy in the formation resulting from scattering and frictional absorption. Therefore, the sonic log responds strongly to porosity and structural changes in the formation and does not require calibration to a known material to measure in-situ physical properties.

In Figure 6, sonic waveform logs are displayed at near (2.44$\mathrm{m})$ and far $(3.05-\mathrm{m})$ receiver separations with positive amplitudes shaded, time as the horizontal axis, and depth from 225 to $250 \mathrm{~m}$ as the vertical axis. Note the range in traveltime and amplitude of the first arrivals with depth and between the two receivers. The waveforms were analyzed to quantify these variations of compressional velocity and amplitude using a modified semblance algorithm (Kimball and Marzetta, 1984; Goldberg et al., 1984a). Semblance is a measure of the coherence of signals calculated for each time lag and normalized by their autocorrelations. Peak semblance occurs when the time lag equals the propagation velocity divided by the receiver offset; it ranges between 0 and 1 for random and perfectly coherent signals, respectively. Time windows were used to compute the semblance and peak amplitude of two periods of the compressional waveforms.

Compressional-wave attenuation was measured from the sonic waveforms by comparing the frequency spectrum of two signals recorded at separated receivers. Operating in the frequency domain, the source signature and geometrical divergence, which are uncertainties in the borehole, were eliminated by the ratio of two spectra. At 2.44- and 3.05-m receiver offsets from the source, the first two periods of the compressional waveform were isolated from the recorded signals and transformed into the frequency domain using a fast Fourier transform algorithm. $P$-wave amplitude spectra may be approximated by exponential decay over short far-field receiver arrays (Goldberg et al., 1984b; Lang et al., 1987). Assuming frequency-independent $Q, Q_{p}$ can be calculated as in the ultrasonic experiment by a linear fit to the logarithm of the spectral ratio times the compressional velocity and divided by the receiver offset.

Figure 7 shows an example of the time-windowed compressional waveforms and their spectral ratio at a midreceiver depth of $238.5 \mathrm{~m}$. The least-squares regression of 14 frequency points, as drawn in this example, was used to calculate $\mathrm{Q}_{p}$ and the regression error for the $90 \%$ confidence interval. Over the 10 - to $20-\mathrm{kHz}$ frequency band, $\mathrm{Q}_{p}$ is 4.7 and the regression error is $21.9 \%$.

At Site 637 , the highly serpentinized and brecciated peridotites typically generated unstable spectral ratios, indicating that scattering and poor energy coupling significantly affect compressional-wave amplitudes. These effects cannot be separated in the frequency domain from the intrinsic attenuation using spectral ratios (Richards and Menke, 1983; Goldberg et al., 1984b). In addition, internal reflections of $P$-waves in the borehole have contributed to the apparent attenuation by conversion to shear energy, which dissipates radially away from the borehole wall (Paillet and Cheng, 1986). Due to these additional extrinsic losses, $\mathrm{Q}_{p}$ values extracted from the sonic waveforms by spectral ratios were variable and generally lower than 23 , the mean of the resonant bar measurements. Hence, attenuation

\section{Time (milliseconds)}

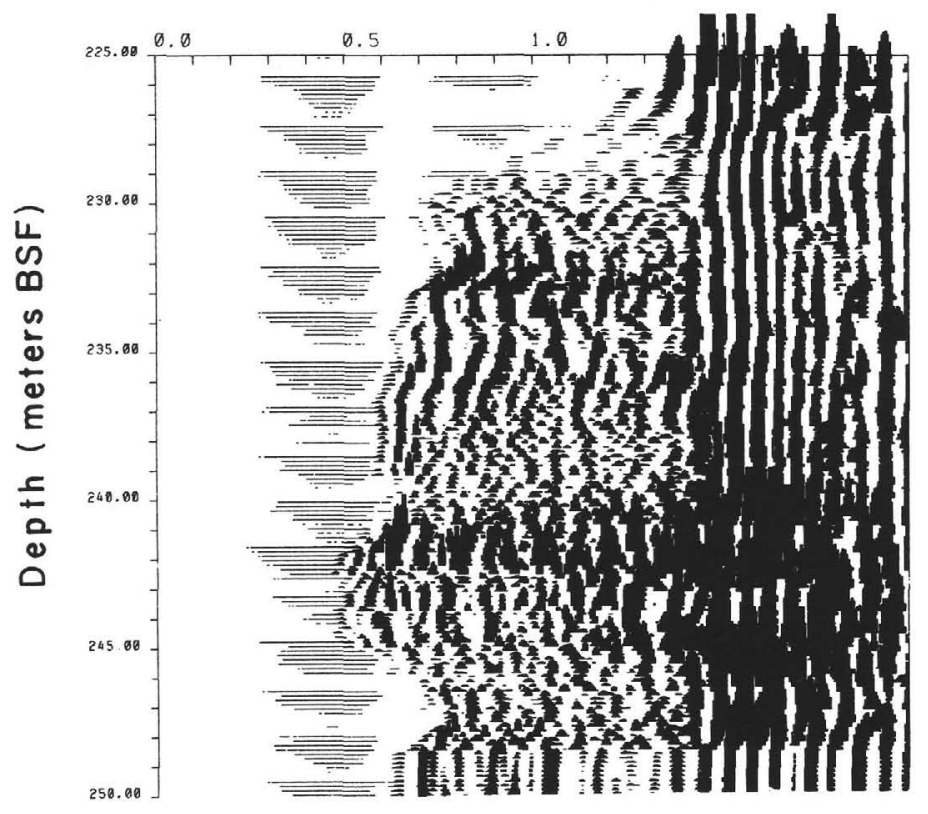

Near Receiver

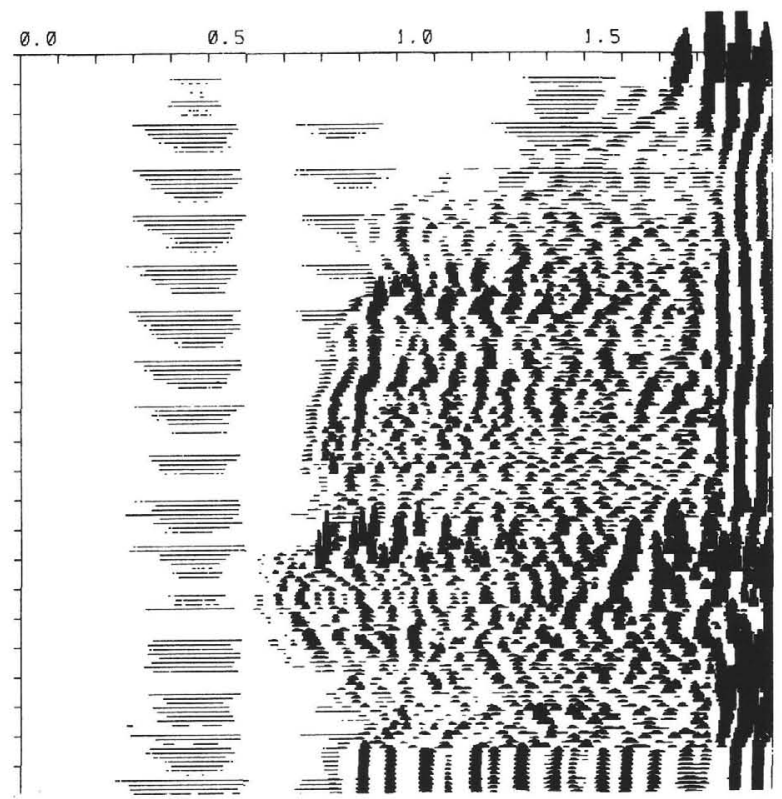

Far Receiver

Figure 6. Sonic waveform logs at near $(2.44-\mathrm{m})$ and far $(3.05-\mathrm{m})$ receiver offsets recorded in altered peridotite from 225 to $250 \mathrm{mbsf}$ in Hole $637 \mathrm{~A}$. The variation in compressional arrival time (first motion) and relative amplitude (shading) over the depth interval are dependent on formation porosity, structure, and composition. Waveform processing for velocity and amplitude can quantify these changes continuously over the depth interval. 

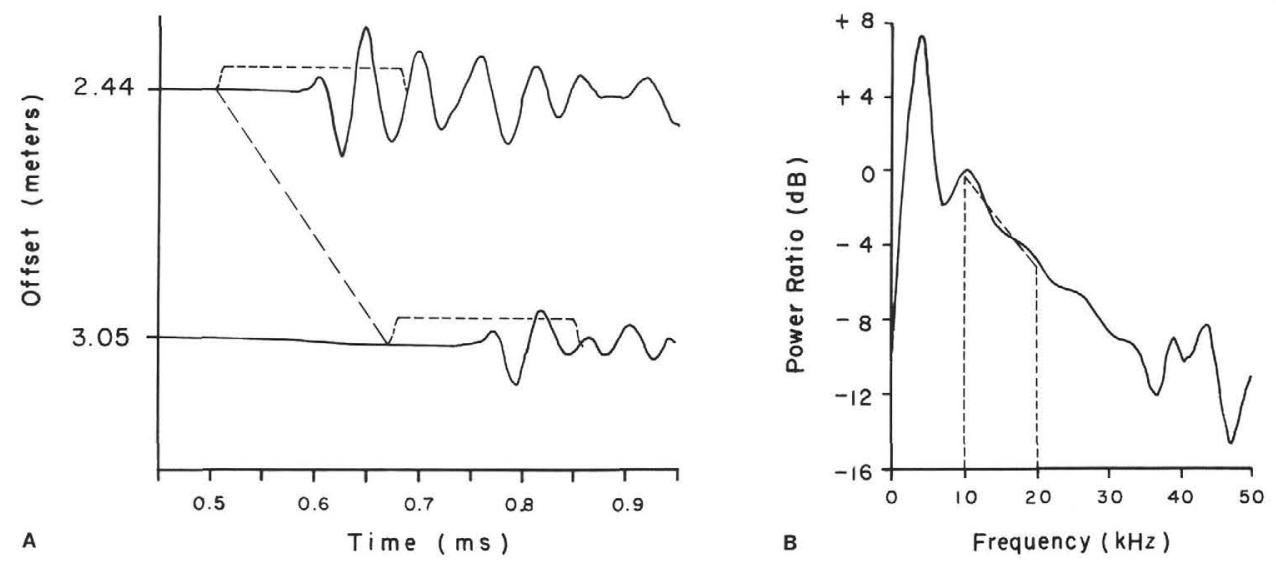

Figure 7. A. Near and far receiver compressional waveforms at a midreceiver depth of $238.5 \mathrm{mbsf}$ in Hole 637A. The moveout between signals corresponds to the compressional velocity. B. Spectral ratio of first two periods of signals shown in Figure 7A. The spectral ratio yields $\mathrm{Q}_{p}=4.7$ in the 10 - to $20-\mathrm{kHz}$ frequency band shown by the regression line (dashed). $\mathrm{Q}_{p}$ values were generally lower than the pressure-compensated resonant bar measurements of $\mathrm{Q}_{p}(23.0 \pm 1.5)$ due to extrinsic losses.

measured directly from sonic waveforms does not reproduce laboratory results, even when measured in the same frequency band, and the extrinsic losses resulted in greater measured in-situ attenuation than resulting from frictional absorption alone. The measurement of in-situ attenuation from sonic waveforms will require corrections for extrinsic effects or measurement by indirect techniques, such as full waveform inversion (e.g., Cheng et al., 1986; Lang et al., 1987).

\section{DISCUSSION}

The velocity, peak semblance, and peak amplitude logs were calculated from the waveforms and are shown in Figure 8. Resonant bar $\mathrm{V}_{p}$ data are also displayed for 6.3-MPa effective pressure, commensurate with the overburden stress in the well. The comparison of resonant bar and $\log$-derived $\mathrm{V}_{p}$ data are in good agreement, surmounting the difficulties of laboratory measurements on impermeable and fragile samples. The estimated experimental error is about $10 \%$, and although greater than the typical 5\% expected for experiments on larger bars of homogeneous rock, it is a reasonable result for these small samples (Winkler, 1979; Bourbié et al., 1987). The differences in laboratory and log-derived velocities may also arise in part from depth uncertainty in the core samples and from large-scale features, such as fractures, that influence the sonic log, but not the laboratory experiments.

The agreement of resonant bar (sonic) laboratory measurements with in-situ sonic log velocity is not biased by the effects of dispersion over a large frequency band, as observed in the comparison of resonant bar and ultrasonic velocities. The measured change in velocity from sonic to ultrasonic frequencies cannot be accounted for simply by a constant-Q model for dispersion, as geometric effects in these small samples probably influenced the ultrasonic velocity. With this in mind, ultrasonic measurements should be interpreted with caution, particularly in conjunction with sonic-logging data, as the differences in frequency and sample size are not correlative. Resonant bar results are generally more reliable, though more time-consuming and limited by sample size and fragility. A better comparison of laboratory and logging acoustic data will require sonic frequency $(10-20-\mathrm{kHz})$ pulse transmission tests in large, solid sample blocks.

The borehole diameter, representative core recovery, and comparative alteration interpreted from the core are also shown in Figure 8 to illustrate relative alteration by leaching and, in particular, the major brecciated zones and intervals cut by calcite veins (Shipboard Scientific Party, 1987; C. A. Evans, pers. comm.,
1986). The 10-in. borehole diameter varies by only about 1 in. and does not correlate with alteration or poor core recovery. Because acoustic waves are strongly dependent on borehole geometry (Paillet and White, 1982), the poor correlation of borehole diameter with peak amplitude suggests strong amplitude control by other changes in structure. Scattering and poor energy coupling across breccias and veins having different elastic moduli than the matrix will decrease the compressional-wave amplitude. The apparent attenuation may therefore be a useful indicator of such zones, particularly where core recovery is poor. As shown in Figure 8, low compressional amplitudes clearly correlate to brecciated zones and calcite veining. Low amplitudes in intervals that were not sampled may be missed breccias or fractures.

Sonic amplitudes have often been correlated with existing fractures (e.g., Morris et al., 1964) and structural features in crystalline rock (Anderson and O'Malley, 1985) but never before correlated with alteration and brecciation in peridotite. It is useful to note that low sonic amplitudes typically correspond to rocks missed (fractures) or poorly sampled (breccias) during coring. In general, better depth-to-core correlation can be obtained in the intervals of poor recovery by interpretation of sonic amplitudes. We recommend that compressional amplitudes be exploited for correlations beyond simple fracturing, as their sensitivity to environmental changes reveals important information that is often unsampled.

\section{CONCLUSIONS}

Laboratory measurements of velocity and attenuation in altered peridotite from Site 637 were made in the ultrasonic (500$\mathrm{kHz}$ ) and the sonic (10- to $20-\mathrm{kHz}$ ) frequency bands under simulated in-situ effective stress of about 6.3 MPa. Compressionalwave velocity and attenuation were also computed from sonic $\log (10-20-\mathrm{kHz})$ waveforms recorded over the same interval in this well. Ultrasonic velocity and attenuation are considerably greater than the resonant bar results due to both dispersion and geometric effects, suggesting that ultrasonic measurements should be used cautiously for correlations with sonic logs. The pressure-compensated resonant bar measurements of $\mathrm{V}_{p}$ are in good agreement with the sonic $\log$, although measurements of $Q_{p}$ are generally greater. This is primarily due to variable extrinsic effects, such as interferences and scattering from boundaries and radiation of shear energy away from the borehole, that cannot be separated from the apparent in-situ attenuation. However, the total amplitude reduction resulting from combined extrinsic and 


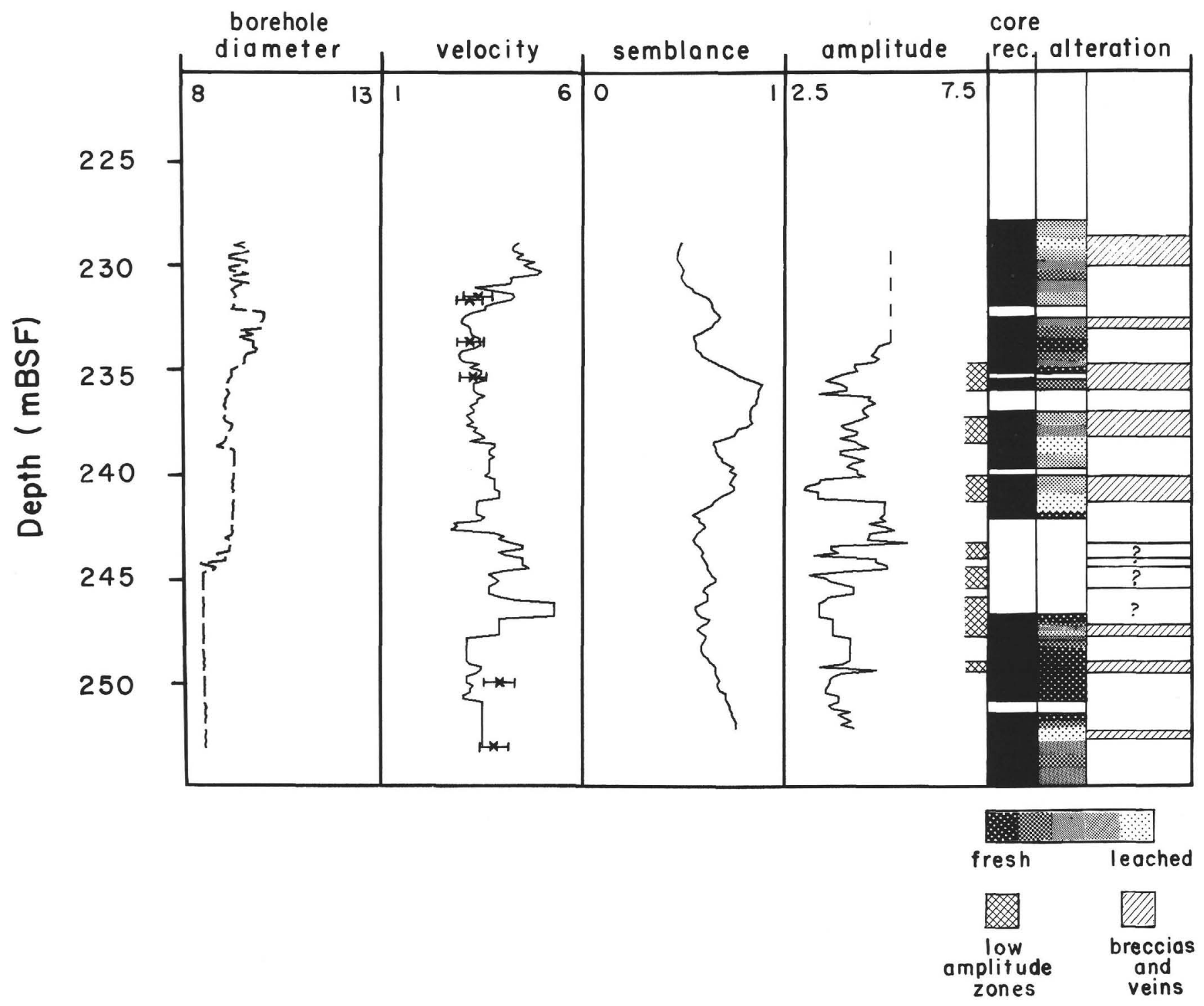

Figure 8. Downhole logs of borehole diameter (in.), compressional velocity ( $\mathrm{km} / \mathrm{s})$, normalized semblance, and amplitude (dB) for altered peridotite drilled at ODP Hole 637A. Laboratory resonance data with $10 \%$ error bars are also shown in the compressional velocity column and are in good agreement with the velocity log. Generalized alteration and core recovery logs from shipboard descriptions are shown for correlation with the compressional amplitude log (see text).

intrinsic losses can be correlated to highly altered and brecciated zones that were poorly sampled during coring of Hole 637A.

\section{ACKNOWLEDGMENTS}

This research was supported by the Joint Oceanographic Institutions and by the Société Nationale de Elf-Aquitaine Production. We greatly appreciated the assistance of the Institut Français du Pétrole. Special thanks are due to T. Bourbié, N. Lucet, M. Mason, L. Nicoletis, P. Rasolofosoan, and P. Tarif for their time and effort in this research. C. Evans assisted in describing the relative core alteration for Figure 8.

\section{REFERENCES}

Anderson, R. N., and O'Malley, H., 1985. Frequency response and attenuation changes across highly altered fracture zones crosscutting a fast formation: the oceanic crust of the Mid-Atlantic Ridge. Trans. SPWLA Annu. Logging Symp., 26:Pap. LLL.

Arons, J., Murray, J., and Seeman, B., 1978. Formation compressional and shear interval transit time logging by means of land spacings and digital techniques. Trans. Soc. Pet. Eng. AIME Annu. Tech. Conf., 53:Pap. 7446.
Biot, M. A., 1956. Theory of elastic waves in a fluid-saturated porous solid, II. Higher frequency range. J. Acoust. Soc. Am., 28:179-191. 1962. Generalized theory of acoustic propagation in porous dissipative media. J. Acoust. Soc. Am., 34:1254-1264.

Birch, F., and Bancroft, D., 1938. The effect of pressure on the rigidity of rocks. J. Geol., 46:113-141.

Born, W. T., 1941. The attenuation constant of earth materials. Geophysics, 6:132-148.

Bourbié, T., Coussy, O., and Zinszner, B., 1987. Acoustics of Porous Media: Paris (Editions Technip).

Cheng, C. H., Wilkens, R. H., and Meredith, J. A., 1986. Modeling of full waveform acoustic logs in soft marine sediments. Trans. SPWLA Annu. Logging Symp., 27:Pap. LL.

Futterman, W. I., 1962. Dispersive body waves. J. Geophys. Res., 67: 5279-5291.

Goldberg, D. S., Gant, W. T., Seigfried, R. W., and Castagna, J. P., 1984a. Processing and interpretation of sonic log waveforms: a case study. Trans. Soc. Explor. Geophys. Annu. Symp., 54:28-31.

Goldberg, D. S., Kan, T. K., and Castagna, J. P., 1984b. Attenuation measurements from sonic log waveforms. Trans. SPWLA Annu. Logging Symp., 25:Pap. NN. 
Kimball, C. V., and Marzetta, T. J., 1984. Semblance processing of borehole acoustic array data. Geophysics, 49:274-281.

Kjartansson, E., 1979. Constant-Q wave propagation and attenuation. J. Geophys. Res., 84:4737-4748.

Kolsky, H., 1956. The propagation of stress pulses in viscoelastic solids. Philos. Mag., 8:693-710.

Lang, S. W., Kurkjian, A. L., McClellan, J. H., Morris, C. F., and Parks, T. W., 1987. Estimating slowness dispersion from arrays of sonic logging waveforms. Geophysics, 52:530-544.

Morris, R. L., Grine, D. R., and Arkfe1d, T. E., 1964. Using compressional and shear acoustic amplitudes for the location of fractures. $J$. Pet. Technol., 16.

Murphy, W. F., 1982. Effects of microstructure and pore fluids on granular sedimentary materials [Ph.D thesis]. Stanford Univ.

Paillet, F. L., and Cheng, C. H., 1986. A numerical investigation of head wave and leaky modes in fluid-filled boreholes. Geophysics, 51:1438-1449.

Paillet, F. L., and White, J. E., 1982. Acoustic modes of propagation in the borehole and their relationship to rock properties. Geophysics, 47:1215-1228.

Papadakis, E. P., 1975. Ultrasonic diffraction from single apertures with application to pulse measurements and crystal physics. Phys. Acoust., 11:151-211.

Peselnick, L., and Zietz, I., 1959. Internal friction of fine-grained limestones at ultrasonic frequencies. Geophysics, 24:285-296.

Pickett, G. R., 1963. Acoustic character logs and their application in formation evaluation. J. Petr. Technol., 15:659-667.

Richards, P. G., and Menke, W., 1983. The apparent attenuation of a scattering medium. Bull. Seismol. Soc. Am., 73:1005-1021.
Schreiber, E., Anderson, O. L., and Soga, N., 1973. Elastic Constants and their Measurements: New York (McGraw-Hill).

Shipboard Scientific Party, 1987. Site 637. In Boillot, G., Winterer, E. L., et al., Proc. ODP, Init. Repts., 103: College Station, TX: (Ocean Drilling Program), 123-219.

Spencer, J. W., 1981. Stress relaxation at low frequencies in fluid-saturated rocks: attenuation and modulus dispersion. J. Geophys. Res., 86:1803-1812.

Tarif, P., 1986. Mesure de l'atténuation des ondes compressionelles ultrasoniques dans les roches: application à la mesure de l'anisotropie d'atténuation [Ph.D these]. Univ. Pierre et Marie Curie, Paris.

White, J. E., 1986. Biot-Gardner theory of extensional waves in porous rods. Geophysics, 51:742-745.

Winkler, K., 1979. The effects of pore fluids and frictional sliding on seismic attenuation [Ph.D thesis]. Stanford Univ.

Winkler, K., and Nur, A., 1979. Pore fluids and seismic attenuation in rocks. Geophys. Res. Lett., 6:1-4.

Winkler, K., and Plona, T. J., 1982. Techniques for measuring ultrasonic velocity and attenuation spectra in rocks under pressure. $J$. Geophys. Res., 87:10776-10780.

Wyllie, M.R.J., Gardner, G.H.F., and Gregory, A. R., 1962. Studies of elastic wave attenuation in porous media. Geophysics, 27:569-589.

Date of initial receipt: 17 March 1987

Date of acceptance: 9 September 1987

Ms 103B-163 